\title{
Pentoxifylline Reduces the Incidence and Severity of Necrotizing Enterocolitis in a Neonatal Rat Model
}

\author{
JAVEED TRAVADI, SANJAY PATOLE, ADRIAN CHARLES, BOHUSLAV DVORAK, DOROTA DOHERTY, AND \\ KAREN SIMMER
}

\begin{abstract}
Women's and Children's Health Service [J.T., S.P., A.C., D.D., KS], Women's and Infants' Research Foundation and The University of Western Australia, Perth, Western Australia, Australia 6008; Department of Pediatrics [B.D.], University of Arizona; Tucson, Arizona
\end{abstract}

\begin{abstract}
Necrotizing enterocolitis (NEC) is a potentially fatal illness in premature neonates. Tumor necrosis factor alpha (TNF- $\alpha$ ) has been shown to play a central role in the inflammatory cascade leading to the development of NEC. Published evidence points to a significant role of pentoxifylline in inhibition of TNF- $\alpha$ and in reducing mucosal injury and improving healing in ischemiareperfusion experiments. Our aim was to investigate the effect of pentoxifylline on the incidence of NEC in a neonatal rat model. Newborn Sprague-Dawley rat pups originating from eight separate litters were delivered by cesarean section at $21.5 \mathrm{~d}$ and were formula fed from birth by orogastric gavage. The rat pups were randomized to receive either intraperitoneal pentoxifylline $(15 \mathrm{mg} / \mathrm{kg} / \mathrm{dose})$ or placebo, given every $8 \mathrm{~h}$ beginning at $24 \mathrm{~h}$ of age, in a blinded fashion. Experimental NEC was induced by exposure to hypoxia for $60 \mathrm{~s}$ followed by cold stress at $4^{\circ} \mathrm{C}$ for $10 \mathrm{~min}$. The animals were euthanized at development of NEC or at $96 \mathrm{~h}$ and intestinal tissue was processed and examined for histologic changes of NEC. The incidence of NEC was significantly lower in the pentoxifylline group [pentoxifylline $5 / 38$ versus placebo $15 / 36 ; p=0.008$, odds ratio $(\mathrm{OR})=0.2195 \%$ confidence interval (CI) 0.07-0.67]. Among the pups developing NEC, significantly fewer rat pups treated with pentoxifylline had severe $(\geq 3)$ intestinal injury scores [pentoxifylline $1 / 5$ versus placebo $10 / 15 ; p=0.031$, OR $0.06,95 \%$ CI $0.01-0.79]$. We conclude that intraperitoneal administration of pentoxifylline significantly reduced the incidence and severity of NEC in our experimental animal model. (Pediatr Res 60: 185-189, 2006)
\end{abstract}

$\mathrm{N}^{\mathrm{E}}$ EC is the commonest gastrointestinal emergency in neonates. The incidence of this potentially disastrous illness $(5-10 \%$ of all neonates $<32$ wk gestation) remains almost unchanged despite the recent advances in neonatal intensive care $(1,2)$. The mortality related to NEC continues to range from $10 \%$ to $50 \%$, and approaches $100 \%$ for those with the most severe form of the disease, involving the entire bowel (3). Significant morbidity includes long-term requirement for parenteral nutrition, recurrent bouts of sepsis, malabsorption and malnutrition related to short bowel syndrome (2), and

Received November 30, 2005; accepted March 16, 2006.

Correspondence: Sanjay Patole, M.D., FRACP, King Edward Memorial Hospital for Women, Bagot Road, Subiaco, WA 6008, Australia; e-mail: Sanjay.Patole@ health.wa.gov.au

J.T. is currently affiliated with John Hunter Children's Hospital, Newcastle, New South Wales, Australia 2305.

This work was funded by a Channel 7 Telethon Research Fellowship and the Women's and Infant's Research Foundation, Perth, WA.

DOI: 10.1203/01.pdr.0000228325.24945.ac neurodevelopmental impairment $(4,5)$, especially in infants requiring surgical intervention (6).

Despite extensive research, the pathophysiology of NEC remains poorly understood. Massive endogenous production of cytokines, including tumor necrosis factor-alpha (TNF- $\alpha$ ), occurs following an initial insult and mild mucosal damage to the gut (7). A synergistic effect of these cytokines triggers a cascade of events leading to the eventual breakdown of the gut mucosal barrier and severe NEC in some cases. TNF- $\alpha$, a monokine produced by mononuclear leukocytes, plays a central role in this process (7). It has proinflammatory actions such as induction of leukocyte and endothelial adhesion molecules, activation of polymorphonuclear (PMN) leukocytes, and production of other cytokines like eicosanoids $(8,9)$, platelet activating factor (PAF), (10) and TNF- $\alpha$ itself $(8,9)$.

Elevated plasma levels of TNF- $\alpha$ derived from circulating mononuclear phagocytes have been demonstrated in neonates with NEC (11). However, these plasma TNF- $\alpha$ concentrations have not been shown to correlate with disease severity $(11,12)$. Elevated levels of TNF- $\alpha$ mRNA levels have also been shown in intestinal samples of infants with acute NEC (13), specifically in Paneth cells located at the base of the crypts of intestinal mucosa and in infiltrating eosinophils and macrophages (14). Recently, Halpern et al. (15) have shown significantly increased hepatic Kupffer cells and TNF- $\alpha$ content in liver of rat pups with NEC, and proposed the liver as a major source of intestinal TNF- $\alpha$. Pentoxifylline is a methylxanthine derivative with diverse immunomodulatory properties including decreased production of TNF- $\alpha$ (16). It has been shown to increase neutrophil deformability and chemotaxis and diminish the degranulation, adherence, and superoxide generation by stimulated neutrophils $(17,18)$. Pentoxifylline exerts these effects on PMN through its properties of nonspecific phosphodiesterase inhibition resulting in a dosedependent increase in intracellular cAMP (19). For TNF- $\alpha$

Abbreviations: NEC, necrotizing enterocolitis; PAF, platelet activating factor; PMN, polymorphonuclear 
primed PMN, pentoxifylline acts by enhancing the effect of adenosine on degranulation and superoxide production (20).

Animal experiments suggest a significant role of pentoxifylline in improving the gut status in presence of hypoxicischemic injury with reduction in myeloperoxidase activity (an index of PMN stimulation) and free radical generation $(21,22)$. Improved anastomotic healing following pentoxifylline treatment has been demonstrated in ischemia-reperfusion models (23). Pentoxifylline has also been shown to improve hemodynamic function and reduce plasma TNF- $\alpha$ levels and mortality in preterm neonates with blood culture-positive sepsis $(24,25)$. Given the current understanding of inflammatory cytokines like TNF- $\alpha$ in the pathophysiology of the illness, pentoxifylline may be beneficial in the management of NEC. We aimed to investigate the efficacy of pentoxifylline in reducing the incidence and severity of NEC in the neonatal rat model originally described by Barlow in 1975 (26). This model incorporates the stress of hypoxia, hypothermia, and artificial feeding on an immature gut (the most essential risk factors for human NEC) and the developmental aspects of the illness and has been shown to closely approximate the clinicopathologic findings of human NEC $(27,28)$.

\section{METHODS}

Animal protocol. The animal protocol was approved by the Animal Ethics Committee at the Royal Perth Hospital (application number 02-04). Timedated pregnant Sprague-Dawley rats (Animal Resources Center, Perth, Western Australia) were delivered prematurely on d 21.5 (term, 22.5-23 d) via abdominal incision under $\mathrm{CO}_{2}$ anesthesia. Newborn rat pups (8-12 per litter) were collected, weighed, warmed, and reared in individual labelled Styrofoam cups lined with shredded tissue paper kept in an incubator at $35.5^{\circ} \mathrm{C}$ with a relative humidity of $70 \%$. The rat pups were hand fed with artificial formula (Rat milk replacer: $168 \mathrm{kcal} / 100 \mathrm{~mL}$; Wombaroo Food Products, Adelaide, Australia), designed to approximate the protein and caloric content of rat breast milk, using an orogastric tube (L-Cath 28-G, Luther Medical Products, Inc., Tustin, CA). Feeds were started at $0.1 \mathrm{~mL}$ from $30 \mathrm{~min}$ of age, given every $4 \mathrm{~h}$, and advanced by $0.05 \mathrm{~mL}$ every $12 \mathrm{~h}$ to a maximum of $0.3 \mathrm{~mL}$ per feed by $\mathrm{d} 4$. Routine care included daily cleansing and stimulation of bowel and bladder function before each feed using a cotton-tipped applicator to simulate maternal grooming behavior. Body weights were recorded daily prefeed. To develop NEC, all rat pups were challenged with hypoxia by breathing $100 \%$ nitrogen gas in a closed, clear plastic chamber for $60 \mathrm{~s}$, followed by hypothermia - exposure to cold at $4^{\circ} \mathrm{C}$ for $10 \mathrm{~min}$ - starting from $2 \mathrm{~h}$ of age and then every $12 \mathrm{~h}$.

Rat pups were randomized to the two experimental groups using computergenerated random numbers stratified for each litter to ensure that rat pups from each litter were evenly distributed in the study groups. Group allocation was determined by selection of sequentially numbered opaque envelopes. Group 1 received $15 \mathrm{mg} / \mathrm{kg}$ of pentoxifylline $(200 \mu \mathrm{g} / 0.1 \mathrm{~mL})$, whereas Group 2 received an equivalent volume of placebo (normal saline). Starting with the first dose at $24 \mathrm{~h}$ of age, both medications were administered intraperitoneally at 8-h intervals using a tuberculin syringe. The study medications were prepared by the Department of Pharmacy at the Princess Margaret Hospital for Children, Perth, Western Australia, and dispensed in de-identified 5-mL vials. All investigators including the histopathologist (AC) and biostatistician (DD) were blinded to study group allocation. The dose of pentoxifylline used in the study was based on data from clinical trials of pentoxifylline in neonatal sepsis and chronic lung disease $(25,29)$ and the intraperitoneal route was chosen for ease of administration.

All rat pups were observed hourly for clinical signs of NEC such as abdominal distension, respiratory distress, and lethargy. Ninety-six hours after birth, or at signs of illness, the pups were euthanized via decapitation.

Tissue collection. Within $30 \mathrm{~min}$ of death, the gastrointestinal tract (stomach to rectum) was harvested and visually evaluated for intestinal discolouration, hemorrhage, and distension. The entire bowel was then fixed in $10 \%$ buffered formalin, embedded in paraffin, and 5- $\mu \mathrm{m}$ sections were stained with hematoxylin and eosin (HE) for microscopic evaluation. The entire bowel was processed as macroscopic changes do not correlate well with microscopy and the disease (as in human neonates) tends to be patchy.

Histopathological evaluation. A perinatal histopathologist (AC), blinded to study group allocation, assessed and graded the histologic changes using intestinal injury scores reported by Dvorak et al. (28) as follows: grade 1: focal mild injury confined to villous tips; grade 2: partial loss of villi; grade 3: necrosis extending to submucosa; grade 4: transmural necrosis.

Statistics. Based on the incidence of NEC (60-65\%) in our laboratory using the neonatal rat model, we estimated a total sample size of 80 animals (40 in each group) to detect a 50\% reduction in the incidence of NEC ( $\alpha 0.05$, $\beta 0.2)$ in the treatment group. Differences between groups for incidence and severity of intestinal injury were compared using the Fisher exact tests. Effect of pentoxifylline on NEC was summarized using odds ratio (OR) and their 95\% confidence intervals (CI) estimated using logistic regression analysis.

\section{RESULTS}

Eighty rat pups originating from eight separate litters were used for the study. Fifty-nine rat pups (pentoxifylline, 35; placebo, 24) survived until $96 \mathrm{~h}$ of age with the remaining animals dying or euthanized on developing clinical signs of illness. The interval between death and tissue harvest was greater than $30 \mathrm{~min}$ in 6 rat pups (pentoxifylline, 2; placebo, 4) and these were excluded (as postmortem autolysis can mimic early NEC changes), leaving 38 in the pentoxifylline and 36 in the placebo group for the final analysis.

The mean \pm SD birth weight of the rat pups in the placebo group was $5.3 \pm 0.4 \mathrm{~g}$, whereas in the pentoxifylline group it was $5.2 \pm 0.4 \mathrm{~g}$. The body weights of rat pups in both the groups gradually declined over the study period, with the final weights being $3.8 \pm 0.3 \mathrm{~g}$ for the placebo and $4.0 \pm 0.3 \mathrm{~g}$ for the rat pups who received pentoxifylline (Fig. 1). This weight decline was more pronounced for rat pups in the placebo group at $96 \mathrm{~h}$ and was statistically significant-SED 0.0953 , 95\% CI 1.28-1.66.

On histopathological examination, a significantly higher number of rat pups in the placebo group revealed changes consistent with NEC-placebo 15/36 (42\%) versus pentoxifylline $5 / 38$ (13\%); $p=0.008, \mathrm{OR}=0.21,95 \%$ CI $0.07-0.67$ (Fig. 2). These changes ranged from loss of the mucosa over

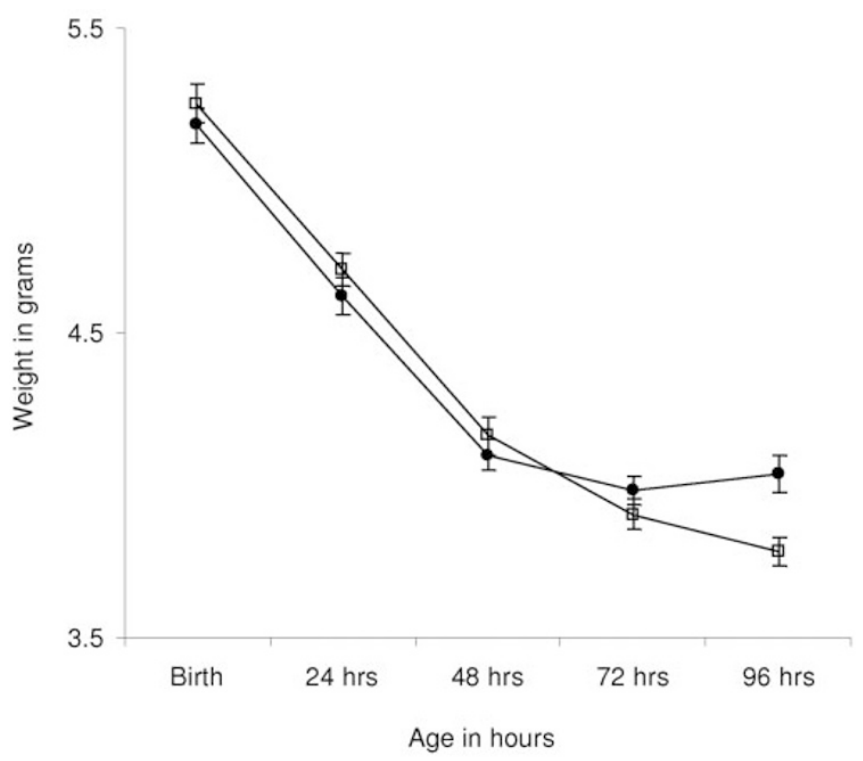

Figure 1. Body weight changes in rat pups in pentoxifylline $(\bullet, n=38)$ and placebo $(\square, n=36)$ groups. Values expressed as mean \pm SEM. 


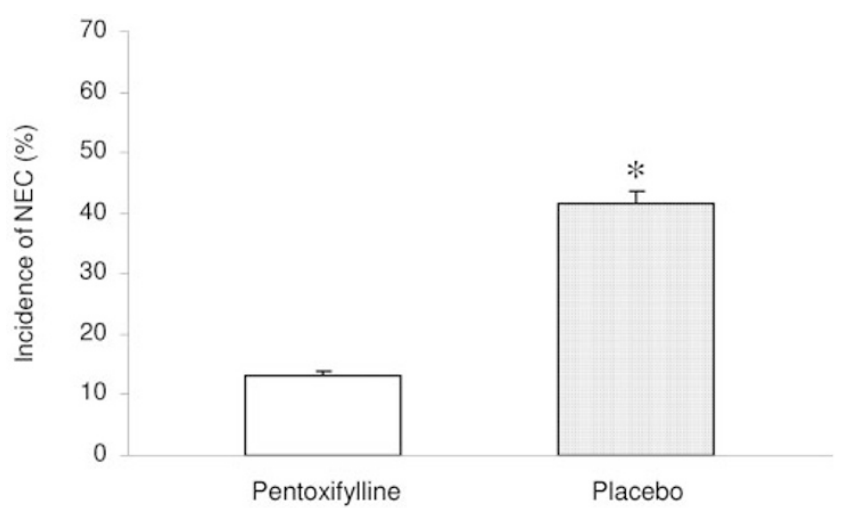

Figure 2. Incidence of NEC. Pentoxifylline group: $n=5 / 38$ (13\%); placebo group: $n=15 / 36(42 \%) .{ }^{*} p=0.008$, OR $=0.21,95 \%$ CI $0.07-0.67$.

the villous tips to loss of villous integrity with villous sloughing, and in late stages degenerative changes of the muscularis propria (Fig. 3). No difference was noted in the distribution of lesions in the animals developing NEC in the two groups. The pathologic changes of NEC were more profound in the rat pups from the placebo group, with animals who developed NEC presenting with more severe $(\geq 3)$ intestinal injury scores-placebo 10/15 (67\%) versus pentoxifylline $1 / 5$ (20\%); $p=0.031$, OR 0.06, 95\% CI 0.01-0.79 (Fig. 4).

\section{DISCUSSION}

Our results indicate a significant reduction in the severity and incidence of NEC in the rat pups treated with pentoxifylline.

Several models for studying NEC in pigs, dogs, rabbits, mice, and rats have been extensively reviewed (30,31). Most of these do not incorporate the common risk factors (e.g. prematurity, hypoxia, hypothermia, and formula feeding) (7) for neonatal NEC and focus on induction of ischemia/ reperfusion injury using chemical or surgical techniques to produce NEC-like pathologic changes. The rat model used in our study is the most representative as it incorporates the currently accepted common risk factors for NEC in human preterm neonates. The results of our study are thus more relevant to NEC in human preterm neonates.

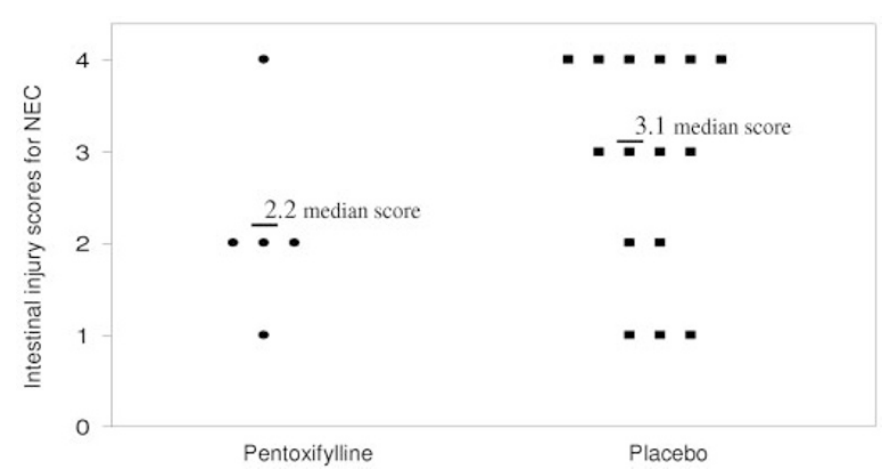

Figure 4. Intestinal injury scores. Grade 1: focal mild injury confined to villous tips; grade 2: partial loss of villi; grade 3: necrosis extending to submucosa; grade 4: transmural necrosis.

The pathophysiology of NEC involves a complex interaction of pro- (IL-6, IL-8, IL-12, IL-18, interferon gamma, TNF- $\alpha$, and PAF) and anti-inflammatory (IL-1ra, IL-6, IL-10, IL-11) mediators $(11,32,33)$. In particular, TNF- $\alpha$ along with PAF has been implicated to play a central role in the inflammatory cascade leading to NEC $(7,11)$. TNF- $\alpha$ triggers the production of several inflammatory cytokines including PAF, is one of the most potent inducers of oxygen radicals in neutrophils and plays an important role in the regulation of intestinal epithelial cell apoptosis $(34,35)$. The beneficial effects of pentoxifylline in our study could be related to the inhibition of TNF- $\alpha$, indirect inhibition of PAF, reduction in free radicals, and activation of leukocytes. The ability of pentoxifylline to improve capillary flow and tissue oxygenation may also have been helpful (36).

The importance of TNF- $\alpha$ in the pathophysiology of NEC is also indicated by two recent experimental studies involving anti-TNF- $\alpha$ antibodies as a prophylaxis for the illness. Seitz et al. (37) in their rabbit model of NEC reported a significant reduction in the severity of NEC (mean NEC score: $1.72 \pm$ 0.41 versus $3.28 \pm 0.32$ ) and number of myeloperoxidase positive cells (mean 34.33/20 visual fields versus mean $65.85 / 20$ visual fields) in animals exposed to prophylactic administration of rabbit anti-mouse TNF- $\alpha$ antibody. Halpern et al. (15) have also recently suggested that anti-TNF- $\alpha$
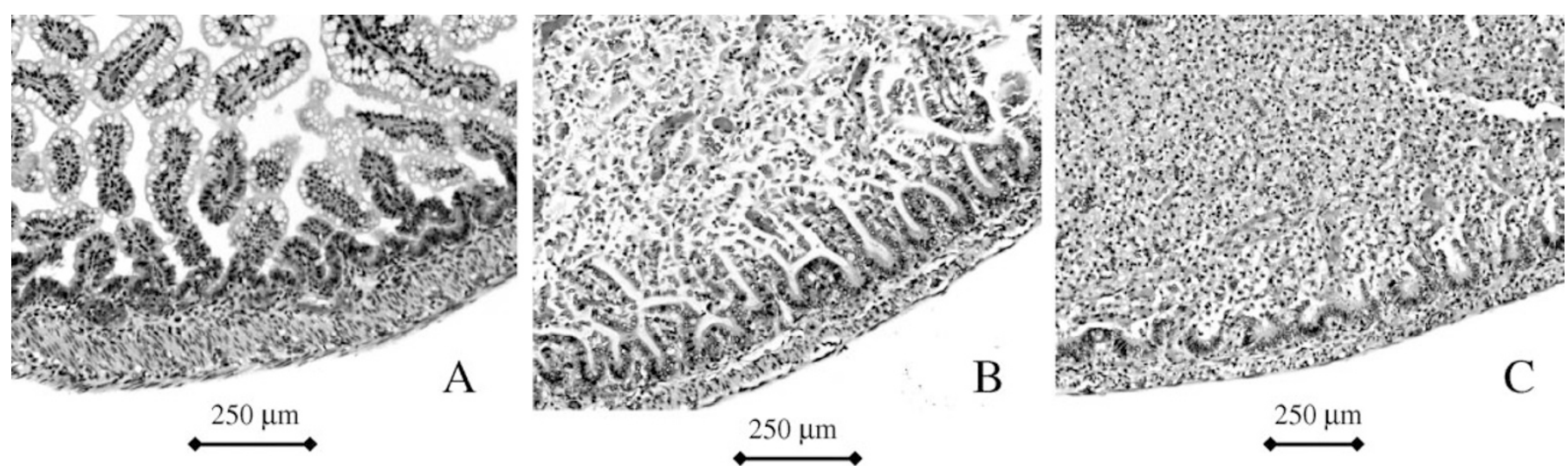

Figure 3. Histopathological appearance of the distal small intestine of neonatal rat pups (H\&E staining). (A) Normal villous appearance. (B) Partial loss of villi with separation in the submucosa and lamina propria. $(C)$ Transmural necrosis with loss of villous and crypt epithelium and infiltration with inflammatory cells. 
antibody could be used as a potential therapy for human NEC and that hepatic TNF- $\alpha$ is an important component for the development of NEC. Using a similar neonatal rat model of NEC, they showed that in animals injected with a monoclonal anti-TNF- $\alpha$ antibody, the incidence of NEC was reduced from $80 \%$ to $17 \%$. Also, hepatic TNF- $\alpha$, and hepatic and ileal IL-18 were significantly decreased in pups given anti-TNF- $\alpha$. However, an increased risk of serious infections and nonHodgkin's lymphoma following immunosuppression due to specific and complete inhibition of TNF- $\alpha$ limits the use of this therapy in human neonates.

The timing of pentoxifylline administration in our study is of interest. The incidence and severity of NEC was reduced in the newborn rat pups when treated with pentoxifylline starting $24 \mathrm{~h}$ after the initiation of stress. Caplan et al. (27) have reported onset of NEC within $36 \mathrm{~h}$ of initiation of stress in the hypoxia-hypothermia-formula-fed preterm rat model of the illness. It is thus possible that pentoxifylline may have acted as a treatment (or secondary prophylaxis) rather than a primary prophylactic agent at least in some pups that had developed early NEC before the drug was administered.

Erdener et al. (38) did not observe such a protective effect of pentoxifylline on NEC in their hypoxia/reperfusion model using 1-d-old newborn rabbits. This model, however, differs substantially from ours in that it utilized a different species and used hypoxia as the only stress factor. Their results are also difficult to interpret due to the small sample size and the lack of sample size estimations. Despite the conclusion that pentoxifylline was not beneficial, a closer look at the data does indicate a trend toward less severe NEC scores for rabbits in the pentoxifylline group (score, $1.85 \pm 0.69$ ) compared with the untreated rabbits (score, $2.42 \pm 0.78$ ).

Currently, there is no specific treatment or secondary prophylaxis for NEC. Once the potentially disastrous illness is diagnosed, the progression and outcome of the disease are difficult to predict and management is purely supportive. Our results indicate that pentoxifylline may be used for secondary prevention, preferably as soon as possible after the diagnosis of NEC. The natural course of the illness is often progressive and multifocal, and it has been hypothesized that, as the sick neonate redistributes the blood, other areas of the bowel become compromised and develop NEC (39). It is only with significant disease or perforation that the process becomes clinically apparent. Treatment at an early stage may therefore prevent progression of the illness, and reduce the length of the bowel that may be severely compromised with necrosis. Given that pentoxifylline has been used in chronic lung disease (29) and sepsis (25) in preterm neonates, and so far no adverse effects have been reported, a clinical trial may be justified based on our results. Caution, however, is warranted, as the concept of down-regulation of the inflammatory response is deceptively simple. The difficulty lies in our inability to determine the nature and dominance of the type of immune response (pro- or anti-inflammatory) at any stage of the illness. The importance of species-specific responses to therapeutic agents also needs to be considered carefully. Pilot studies are necessary for evaluating the pharmacokinetics, dose response, and safety of pentoxifylline in preterm neonates. The existing studies of pentoxifylline in critically ill preterm neonates with sepsis should provide useful baseline data to design such pilot studies (40). Clinical trials must also address the issue of long-term neurodevelopmental outcomes. This is especially important for drugs chosen for prophylaxis, as almost an entire population of high-risk preterm neonates will be exposed to their adverse effects.

Acknowledgments. The authors thank Dr. David Pass at the State Animal Resources Center, Western Australia, and Dr. Linda Manning and her staff at the Animal Facility at Royal Perth Hospital for their support and assistance with maintaining the animals. We also thank Brian Rich, Wombaroo Food Products, Adelaide, South Australia, for providing the rat milk substitute formula used in the study.

\section{REFERENCES}

1. Llanos AR, Moss ME, Pinzon MC, Dye T, Sinkin RA, Kendig JW 2002 Epidemiology of neonatal necrotising enterocolitis: a population-based study. Paediatr Perinat Epidemiol 16:342-349

2. Kliegman RM, Fanaroff AA 1984 Necrotizing enterocolitis. N Engl J Med 310:1093-1103

3. Rowe MI, Reblock KK, Kurkchubasche AG, Healey PJ 1994 Necrotizing enterocolitis in the extremely low birth weight infant. J Pediatr Surg 29:987-990

4. Vohr BR, Wright LL, Dusick AM, Mele L, Verter J, Steichen JJ, Simon NP, Wilson DC, Broyles S, Bauer CR, Delaney-Black V, Yolton KA, Fleisher BE, Papile LA, Kaplan MD 2000 Neurodevelopmental and Functional Outcomes of Extremely Low Birth Weight Infants in the National Institute of Child Health and Human Development Neonatal Research Network, 1993-1994. Pediatrics 105:1216-1226

5. Bedrick AD 2004 Necrotizing enterocolitis: neurodevelopmental "risky business". J Perinatol 24:531-533

6. Tobiansky R, Lui K, Roberts S, Veddovi M 1995 Neurodevelopmental outcome in very low birthweight infants with necrotizing enterocolitis requiring surgery. J Paediatr Child Health 31:233-236

7. Hsueh W, Caplan MS, Qu XW, Tan XD, DePlaen IG, Gonzalez-Crussi F 2003 Neonatal necrotizing enterocolitis: clinical considerations and pathogenetic concepts. Pediatr Dev Pathol 6:6-23

8. Jaattela M 1991 Biologic activities and mechanisms of action of tumor necrosis factor-alpha/cachectin. Lab Invest 64:724-742

9. Vilcek J, Lee TH 1991 Tumor necrosis factor. New insights into the molecular mechanisms of its multiple actions. J Biol Chem 266:7313-7316

10. Camussi G, Bussolino F, Salvidio G, Baglioni C 1987 Tumor necrosis factor/ cachectin stimulates peritoneal macrophages, polymorphonuclear neutrophils, and vascular endothelial cells to synthesize and release platelet-activating factor. J Exp Med 166:1390-1404

11. Caplan MS, Sun XM, Hseuh W, Hageman JR 1990 Role of platelet activating factor and tumor necrosis factor-alpha in neonatal necrotizing enterocolitis. J Pediatr 116:960-964

12. Morecroft JA, Spitz L, Hamilton PA, Holmes SJ 1994 Plasma cytokine levels in necrotizing enterocolitis. Acta Paediatr Suppl 396:18-20

13. Viscardi RM, Lyon NH, Sun CC, Hebel JR, Hasday JD 1997 Inflammatory cytokine mRNAs in surgical specimens of necrotizing enterocolitis and normal newborn intestine. Pediatr Pathol Lab Med 17:547-559

14. Tan X, Hsueh W, Gonzalez-Crussi F 1993 Cellular localization of tumor necrosis factor (TNF)-alpha transcripts in normal bowel and in necrotizing enterocolitis. TNF gene expression by Paneth cells, intestinal eosinophils, and macrophages. Am J Pathol 142:1858-1865

15. Halpern MD, Clark JA, Saunders TA, Doelle SM, Husseini DM, Stagner AM, Dvorak B 2006 Reduction of experimental necrotizing enterocolitis with anti-TNF\{alpha\}. Am J Physiol Gastrointest Liver Physiol 290:G757-G764

16. Ward A, Clissold SP 1987 Pentoxifylline. A review of its pharmacodynamic and pharmacokinetic properties, and its therapeutic efficacy. Drugs 34:50-97

17. Krause PJ, Maderazo EG, Contrino J, Eisenfeld L, Herson VC, Greca N, Bannon P, Kreutzer DL 1991 Modulation of neonatal neutrophil function by pentoxifylline. Pediatr Res 29:123-127

18. Currie MS, Rao KM, Padmanabhan J, Jones A, Crawford J, Cohen HJ 1990 Stimulus-specific effects of pentoxifylline on neutrophil CR3 expression, degranulation, and superoxide production. J Leukoc Biol 47:244-250

19. Chilcoat CD, Rowlingson KA, Jones SL 2002 The effects of cAMP modulation upon the adhesion and respiratory burst activity of immune complex-stimulated equine neutrophils. Vet Immunol Immunopathol 88:65-77

20. Sullivan GW, Luong LS, Carper HT, Barnes RC, Mandell GL 1995 Methylxanthines with adenosine alter TNF alpha-primed PMN activation. Immunopharmacology 31:19-29 
21. Sener G, Akgun U, Satiroglu H, Topaloglu U, Keyer-Uysal M 2001 The effect of pentoxifylline on intestinal ischemia/reperfusion injury. Fundam Clin Pharmacol 15:19-22

22. Savas C, Aras T, Cakmak M, Bilgehan A, Ataoglu O, Turkozkan N, Ozguner F, Yucesan S, Dindar H 1997 Pentoxifylline inhibits overflow and reduces intestinal reperfusion injury. J Pediatr Surg 32:905-910

23. Mustafa NA, Yandi M, Albayrak L, Yildiz K 1995 Effect of pentoxifylline on the ischemia-reperfusion injury of the intestine. Int Surg 80:152-155

24. Lauterbach R, Zembala M 1996 Pentoxifylline reduces plasma tumour necrosis factoralpha concentration in premature infants with sepsis. Eur J Pediatr 155:404-409

25. Lauterbach R, Pawlik D, Kowalczyk D, Ksycinski W, Helwich E, Zembala M 1999 Effect of the immunomodulating agent, pentoxifylline, in the treatment of sepsis in prematurely delivered infants: a placebo-controlled, double-blind trial. Crit Care Med 27:807-814

26. Barlow B, Santulli TV 1975 Importance of multiple episodes of hypoxia or cold stress on the development of enterocolitis in an animal model. Surgery 77:687-690

27. Caplan MS, Hedlund E, Adler L, Hsueh W 1994 Role of asphyxia and feeding in a neonatal rat model of necrotizing enterocolitis. Pediatr Pathol 14:1017-1028

28. Dvorak B, Halpern MD, Holubec H, Williams CS, McWilliam DL, Dominguez JA, Stepankova R, Payne CM, McCuskey RS 2002 Epidermal growth factor reduces the development of necrotizing enterocolitis in a neonatal rat model. Am J Physiol Gastrointest Liver Physiol 282:G156-G164

29. Lauterbach R, Pawlik D, Zembala M, Szymura-Oleksiak J, Lisowska-Miszczyk I, Kowalczyk D, Bury J 2004 Pentoxifylline in prevention and treatment of chronic lung disease. Acta Paediatr Suppl 93:20-22

30. Crissinger KD 1995 Animal models of necrotizing enterocolitis. J Pediatr Gastroenterol Nutr 20:17-22
31. Topalian SL, Ziegler MM 1984 Necrotizing enterocolitis: a review of animal models. J Surg Res 37:320-336

32. Edelson MB, Bagwell CE, Rozycki HJ 1999 Circulating pro- and counterinflammatory cytokine levels and severity in necrotizing enterocolitis. Pediatrics 103:766771

33. Halpern MD, Holubec H, Dominguez JA, Williams CS, Meza YG, McWilliam DL, Payne CM, McCuskey RS, Besselsen DG, Dvorak B 2002 Up-regulation of IL-18 and IL-12 in the ileum of neonatal rats with necrotizing enterocolitis. Pediatr Res 51:733-739

34. Strieter RM, Kunkel SL, Bone RC 1993 Role of tumor necrosis factor-alpha in disease states and inflammation. Crit Care Med 21:S447-S463

35. Zabel P, Schade FU 1994 Pentoxifylline and tumour necrosis factor-induced lung injury. Eur Respir J 7:1389-1391

36. Steeb GD, Wilson MA, Garrison RN 1992 Pentoxifylline preserves small-intestine micro-vascular blood flow during bacteremia. Surgery 112:756-763

37. Seitz G, Warmann SW, Guglielmetti A, Heitmann H, Ruck P, Kreis ME, Fuchs J 2005 Protective effect of tumor necrosis factor alpha antibody on experimental necrotizing enterocolitis in the rat. J Pediatr Surg 40:1440-1445

38. Erdener D, Bakirtas F, Alkanat M, Mutaf I, Habif S, Bayindir O 2004 Pentoxifylline does not prevent hypoxia/reoxygenation-induced necrotizing enterocolitis. An experimental study. Biol Neonate 86:29-33

39. Richter A, Gortner L, Moller JC, Tegtmeyer FK 1993 Pathogenetic concepts of neonatal necrotizing enterocolitis. Klin Padiatr 205:317-324

40. Szymura-Oleksiak J, Bury J, Lauterbach R, Pawlowski M 1997 Serum concentrations of pentoxifylline and its metabolites in premature infants with sepsis when administered by continuous intravenous infusion. Pharm Sci 3:1-5 